\title{
RANGE OF MOTION AND PAIN INTENSITY OF THE FIRST METATARSOPHALANGEAL JOINT IN WOMEN WITH HALLUX VALGUS DEFORMA- TION AFTER TWO-MONTH HOME EXERCISE PROGRAMME
}

\author{
Aire Arge $^{1}$, Aleks Lenzner ${ }^{2}$, Helena Gapeyeva ${ }^{1}$, Mati PäÄsuke ${ }^{1}$ \\ ${ }^{1}$ Institute of Exercise Biology and Physiotherapy, University of Tartu, Tartu, Estonia \\ ${ }^{2}$ Department of Orthopaedics and Traumatology, Tartu University Hospital, Tartu, \\ Estonia
}

\begin{abstract}
Hallux Valgus (HV) (also Hallux abducto valgus) is a common deformity of the foot, which causes pain, inflammation and decreased joint mobility in the big toe. There are no universally accepted protocol for treating advanced phase $H V$, corrective surgery seems to be the most common direction of curing advanced $\mathrm{HV}$ deformation. The purpose of this study was to assess the effects of twomonth home exercise programme (HEP) on joint mobility and pain of the first metatarsophalangeal joint (MTP-joint) in women with advanced phase $H V$ deformation. Seven women with mean age $55.6 \pm 2.9$ years with advanced phase $\mathrm{HV}$ deformation (first MTP-joint angle more than 20 degrees) participated in the study. Foot pain in different conditions was assessed with modified Foot Function Index's (FFI) pain subscale before and after HEP. Passive range of motion (PROM) of flexion and extension of the first MTP-joint was measured before and after HEP using a standard mechanical goniometer. A significant increase $(p<0.001)$ in the passive extension of the first MTP-joint was noted after HEP compared with the joint mobility before HEP, also passive flexion increased considerably but no significant difference was noted $(p>0.05)$. Foot pain score decreased significantly $(\mathrm{p}<0.05)$ after HEP. In conclusion, in advanced phase $H V$ two-month HEP was an effective therapeutic approach for increasing first MTP-joint mobility and reducing foot pain.
\end{abstract}

Keywords: Hallux valgus, home exercise programme, passive range of motion, foot pain 


\section{INTRODUCTION}

Hallux Valgus deformation ( $H V)$ is multifactorial deformation of the foot where the first metatarsal bone has deviated medially in regards to the midline of the body and the proximal and distal phalanxes have deviated laterally. Resulting from previous there are changes in position of the foot muscles [2, $8]$. The deformation is considered to be progressive and irreversible $[4,10]$. According to different sources $H V$ occurs in about $23 \%$ of population [7] and is associated with female gender, older age, and pain in other bodily regions [9]. Some authors refer narrow shoes and high heels as a big promoter for developing $H V$ [6]. In mild cases the problem can be only cosmetic, but in more advanced phase there can be pathological changes in gait and plantar pressure, limitations of range of motion (ROM) in foot joints, pain during movement and walking and problems in regards of choosing comfortable shoes [2]. In case of mild to moderate deformation the most common recommendations are to use orthoses; patient is educated about proper footwear and instructed combining orthosis with special exercise and taping, what could alleviate or reduce the symptoms in moderate cases of $H V$ deformation [2]. In advanced phase of $H V$ corrective surgery is mostly indicated [11]. Surgical osteotomy is found to be an effective treatment for painful advanced phase $H V$, while orthoses provide short-term symptomatic relief [13].

The intervention of physical therapy in the advanced phase of $H V$ is considered important mainly in postoperative stage [11]. A few studies are performed to elucidate the effect of physical exercises on the function of foot joints and pain intensity in the advanced phase of $H V$ deformation preoperatively. The purpose of the present study was to compare the range of motion and pain in the first metatarsophalangeal joint (MTP-joint) before and after two-month individual exercise program in women with advanced $\mathrm{HV}$ deformation.

\section{MATERIAL AND METHODS}

\section{Subjects}

Seven female patients aged 50 to 65 years with advanced $H V$ deformation (Hallux Valgus angle (HVA) more than 20 degrees) and who had appointed to corrective surgery in the next six months volunteered to participate in the study. The mean \pm SE age of the patients was $55.6 \pm 2.9$ years, body mass $64.7 \pm 10.5 \mathrm{~kg}$, and BMI $24.4 \pm 2.6 \mathrm{~kg} / \mathrm{m}^{2}$. The subjects were scheduled to corrective surgery and were evaluated by orthopedic surgeon in the Department of Orthopaedics and Traumatology of Tartu University Hospital. The exclusion criteria included overlapped toes, balance impairments, rheumatoid arthritis and other etiology 
arthritis, as well as other orthopedic and neurologic diseases, bone fractures in the last year, cognitive disorders and lack of time for the exercise performance. Before the beginning of the testing session, all subjects completed an informed consent form. The study carried the approval of the Ethics Committee of the University of Tartu. All examinations were performed in the Laboratory of Kinesiology and Biomechanics at the University of Tartu twice - before and after the two-month performance of home exercise program (HEP).

\section{Clinical assessment}

The subjects' clinical assessment was performed by orthopedic surgeon in the Department of Orthopaedics and Traumatology of Tartu University Hospital. First MTP-joint angle (metatarsophalangeal, HVA) and intermetatarsal (IMA) angle in X-ray imaging of Hallux Valgus foot were calculated (Figure 1A). Reference values for healthy subject are HVA less than 15 degrees and IMA less than 9 degrees [7]. Patients with advanced HV deformation with HVA of 20 to 40 degrees and IMA of 10 to 15 degrees were recommended for the present study.

\section{Pain questionnaire}

For subjective assessment of the foot function modified Foot Function Index (FFI) was used. It consists of three subscales - pain, disability and activity limitations [1]. Foot pain was assessed on a 10-point scale in nine different conditions - pain at maximal level, pain in the morning, pain while walking and standing barefoot, pain while walking and standing with shoes, pain while walking and standing with orthosis and pain at the end of the day. The maximum score was 90 points ( $\mathrm{p}$ ), which indicates the worst condition.

\section{Joint mobility}

To measure the passive range of motion (PROM) of the first MTP-joint standard mechanic goniometer was used (Whitehall Manufacturing, USA). The subject was seated on the examination table with feet hanging over the edge, ankle joints in a free position. The axis of the goniometer was placed medially of the first MTP-joint, the proximal end of the goniometer was aligned with the first metatarsal bone and the distal end of the goniometer was aligned with the first proximal phalanx [5]. The first MTP-joint was flexed and extended passively to a maximum range of motion or to the threshold of pain and the reading was fixed (Figure 1B). The measurements were performed three times and best result was documented. 
A
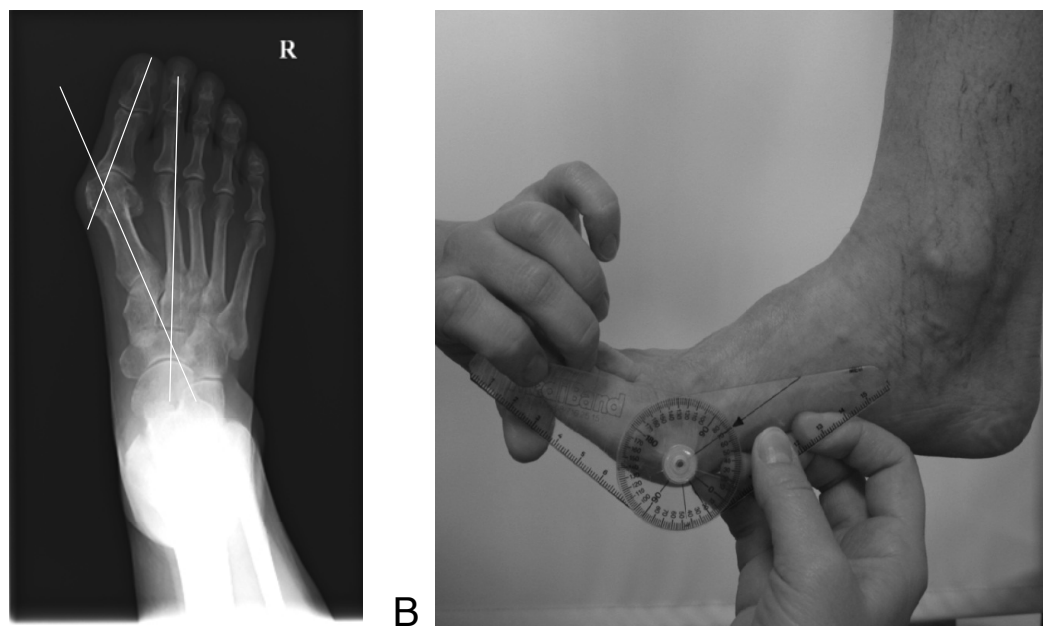

Figure 1. Measurement of first metatarsophalangeal joint angle and intermetatarsal angle in X-ray imaging of Hallux Valgus foot of right leg (A), and of passive range of motion of the first MTP joint flexion (B).

\section{Exercise programme}

HEP consisted of fifteen special exercises, which were derived from combining the elements of postoperative and mild phase $H V$ physiotherapy. The HEP was oriented to improve mobility ( 9 exercises out of 15$)$ and proprioception $(6$ exercises out of 15) of the foot and to strengthen the foot muscles ( 3 exercises out of 15) $[2,11]$. At the first meeting the physiotherapist (AA) explained the exercise programme to subjects, gave them the printed exercise programme with pictures and explanations as well as the exercise diary. In the diary they recorded how many times per day they did each exercise, training time, perception of pain before and after the HEP, perceived intensity of the exercise and fatigue during the exercises. To assess fatigue modified Borg scale was used. Subjects preformed HEP twice a day during two months, average exercise time was 20 minutes.

\section{Statistical analysis}

Results are expressed as means and standard errors $( \pm S D)$. Student paired $t$-test was used to evaluate differences in the results of measurements before and after HEP. A level of $\mathrm{p}<0.05$ was selected to indicate statistical significance. 


\section{RESULTS}

The PROM of the first MTP-joint flexion of the affected foot increased 4.3 degrees $(29 \%)$ and extension increased 17.1 degrees $(39 \%, \mathrm{p}<0.001)$ after two months of HEP (Figure 2). Minimum values of PROM of flexion and extension before HEP were 5 degrees and 35 degrees, respectively, maximum values were 30 degrees and 60 degrees, respectively. Minimum values of PROM of flexion and extension after HEP were 10 degrees and 50 degrees, respectively, maximum values were 30 degrees and 70 degrees respectively. According to Shereff et al [12] the reference values of the PROM of flexion and extension of the first MTP-joint are 34 degrees and 76 degrees, respectively. As compared with reference data the values of PROM of flexion and extension of the first MTP-joint in subjects of the present study with advanced phase $H V$ deformation were significantly lower before HEP and increased to the minimum level of the reference values two months after starting HEP.

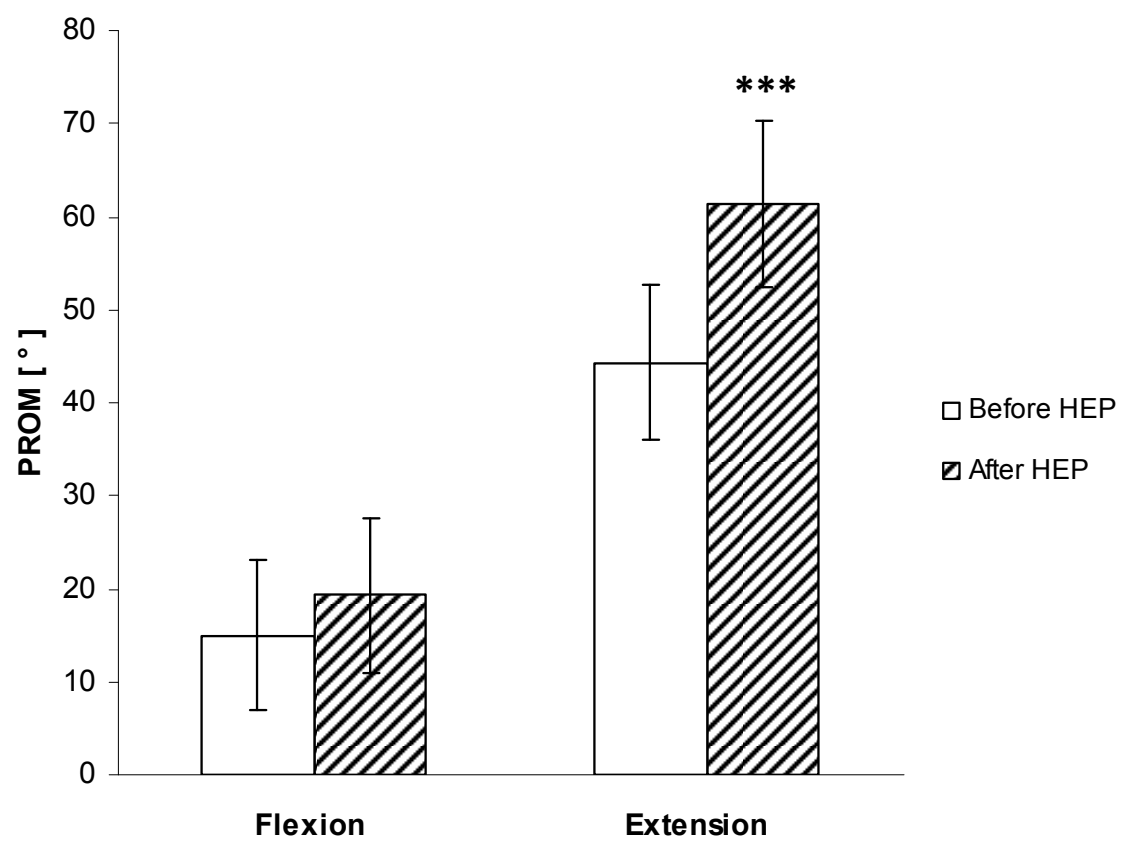

Figure 2. Passive range of motion (PROM) of the first metatarsophalangeal joint flexion and extension before and after two-month home exercise programme (HEP) in women with Hallux Valgus deformation (mean $\pm S E$ ). ${ }^{* * *} p<0.001$ - significant difference in the PROM extension between pre- and post HEP 
Subjects perceived the pain related to the $H V$ deformation in very different levels of intensity. The lowest score on the pain subscale of the FFI at the first assessment was $22 \mathrm{p}$, the highest score was $78 \mathrm{p}$ (the maximum score of FFI pain subscale is $90 \mathrm{p}$ ). After the HEP the lowest score of the FFI pain subscale was $9 \mathrm{p}$, highest score was $48 \mathrm{p}$. The FFI pain subscale average score in the first assessment was $43.1 \mathrm{p}$ and after HEP $22.4 \mathrm{p}$ - pain decreased on an average of $48 \%(\mathrm{p}=0.03)$ (Figure 3).

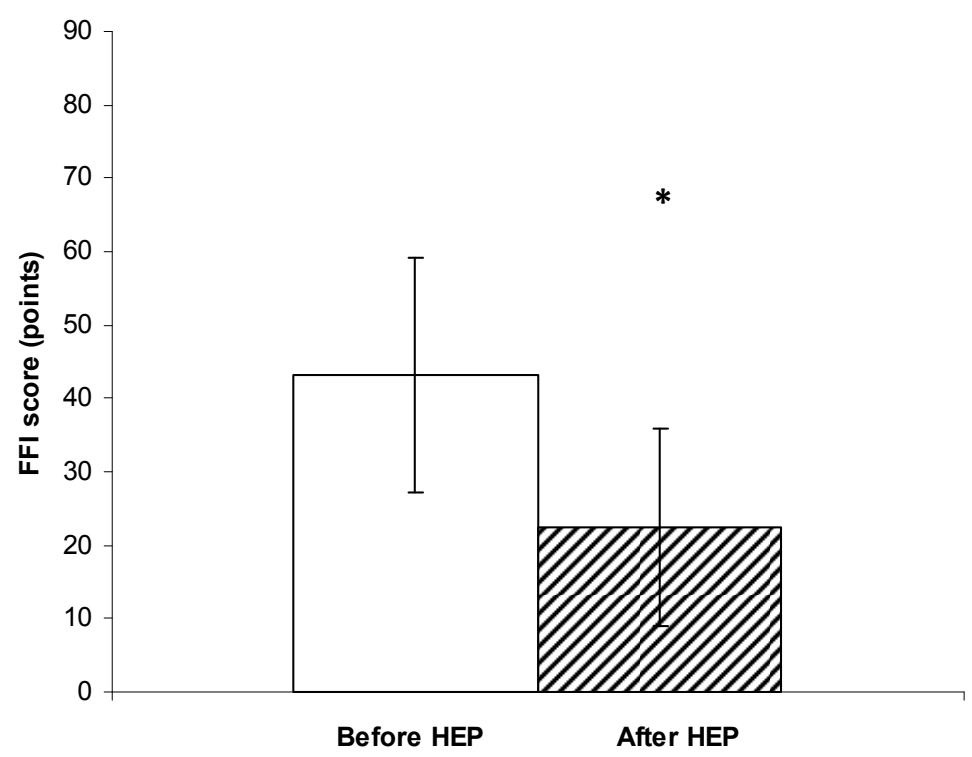

Figure 3. Foot Function Index questionnaire pain intensity score of the first metatarsophalangeal joint before and after two-month home exercise programme in women with Hallux Valgus deformation (mean $\pm \mathrm{SE}$ ). ${ }^{*} \mathrm{p}<0.05$

\section{DISCUSSION}

The present study compared the range of motion and pain in the first MTP-joint before and after two-month individual exercise program in women with advanced $\mathrm{HV}$ deformation. The main findings of the present study were: 1) the PROM of extension of the first MTP-joint increased significantly in women with advanced phase $H V$ deformation after two months of HEP and it reached the first MTP-joint reference value; and 2) women with advanced phase of $\mathrm{HV}$ deformation reported pain intensity decrease of first MTP-joint after two months of HEP.

In mild phases of $H V$ deformation physiotherapy, it is recommended to use orthosis combined with exercises and choosing proper footwear [2], 
postoperatively lymphatic drainage, cold applications, gait training, mobilization of the MTP-joint, strengthening exercises of the flexors and extensors of toes and manual therapy are applied [10]. In this study both the elements of preoperative and postoperative physiotherapy of $H V$ deformation were used [2, 10]. All subjects were very motivated, performed the programme consistently during two months and documented their progress to exercise diary. It was definitely important that women who participated in this study were very interested and participated in the exercise programme regularly.

HV corrective surgery does not always provide total improvement and post-surgery complications may take a long time to recover and surgery is not suitable for every HV patient, so there is a need for studies to test other conservative treatments like exercise programmes, manual therapy, taping and combinations of the previous [3].

In conclusion, this study demonstrated that two-month HEP had a positive effect on foot joint mobility and pain relieving in advanced phase $H V$ deformation. It can be recommended to use home exercise programmes in the advanced phase of $H V$ deformation before corrective surgery.

\section{ACKNOWLEDGEMENTS}

This study was supported by Estonian Ministry of Education and Research project No SF0180030s07 and Estonian Science Foundation project No 7939.

\section{REFERENCES}

1. Agel J, Beskin JL, Brage M, Guyton GP. Kadel NJ, Saltzman CL, Sands AK, Sangeorzan BJ, SooHoo NF, Stroud CC, Thordarson DB. (2005) Reliability of the Foot Function Index: A report of the AOFAS outcomes committee. Foot Ankle Intern, 26: 962-967

2. Bayar B, Erel S, Şimşek IE, Sümer E, Bayar K. (2011) The effects of taping and foot exercises on patients with hallux valgus: a preliminary study. TurkJ Med Sci, 41: 403-409

3. du Plessis M, Zipfel B, Brantingham JW, Parkin-Smith GF, Birdsey P, Globe G, Cassa TK. (2011) Manual and manipulative therapy compared to night splint for symptomatic hallux abducto valgus: an exploratory randomised clinical trial. Foot, 21: 71-78

4. Glasoe WM, Nuckley DJ, Ludewig PM. (2010) Hallux Valgus and the first metatarsal arch segment: A theoretical biomechanical perspective. Phys Ther, 90: $110-120$ 
5. Jones AM, Curran SA. (2012) Intrarater and interrater reliability of first metatarsophalangeal joint dorsiflexion: goniometry versus visual estimation. J Am Podiatr Med Assoc, 102: 290-298

6. Mafart B. (2007) Hallux valgus in a historical French population: Paleopathological study of 605 first metatarsal bones. Joint Bone Spine, 74: 166-170

7. Magee DJ. (2006) Orthopedic Physical Assessment. $4^{\text {th }}$ Edition, Edmonton: Saunders

8. Menz HB, Fotoohabadi MR, Wee E, Spink MJ. (2010) Validity of self-assessment of hallux valgus using the Manchester scale. BMC Musculoskelet Dis, 11: 1-6

9. Menz HB, Roddy E, Thomas E, Croft PR. (2011) Impact of hallux valgus severity on general and foot-specific health-related quality of life. Arthritis Care Res, 63: 396-404

10. Nix S, Smith M, Vicenzino B. (2010) Prevalence of hallux valgus in the general population: a systematic review and metaanalysis. J Foot Ankle Res, 3: 1-9

11. Schuh R, Hofstaetter SG, Adams Jr SB, Pichler F, Kristen K-H, Trnka H-J. (2009) Rehabilitation after Hallux Valgus surgery: importance of physical therapy to restore weight bearing of the first ray during the stance phase. Phys Ther, 89: 934-945

12. Shereff MJ, Bejjani FJ, Kummer FJ. (1986) Kinematics of the first metatarsophalangeal joint. J Bone Joint Surg, 68: 392-398

13. Torkki M, Malmivaara A, Seitsalo S, Hoikka V, Laippala P, Paavolainen P. (2001) Surgery vs orthosis vs watchful waiting for hallux valgus: a randomized controlled trial. J Am Med Assoc, 285: 2474-2480

\section{Correspondence to:}

Aire Arge

Institute of Exercise Biology and Physiotherapy

Faculty of Exercise and Sport Sciences

University of Tartu

E-mail: aireaku@gmail.com

Telephone: (+372) 7376286

Fax: (+372) 7376286 